\title{
The ceramide pathway is involved in the survival, apoptosis and exosome functions of human multiple myeloma cells in vitro
}

\author{
Qian CHENG ${ }^{1, \#}$, Xin $\mathrm{LI}^{1, \#}$, Yue WANG ${ }^{2}$, Min DONG ${ }^{2}$, Feng-huang ZHAN ${ }^{3}$, Jing LIU ${ }^{1, *}$ \\ ${ }^{1}$ Department of Hematology, the Third Xiangya Hospital of Central South University, Changsha 412000, China; ${ }^{2}$ Department of \\ Hematology, the Affiliated Hospital of Guilin Medical College, Guilin 541000, China; ${ }^{3}$ Department of Medicine, Division of Hematology, \\ University of lowa, lowa City, IA 52404, USA
}

\begin{abstract}
Multiple myeloma (MM) is characterized by the clonal proliferation of malignant plasma cells and refractoriness to traditional therapies. It has been shown that exosomes are involved in modulating the progression and the metastasis of cancers through microRNAs (miRs). Ceramide is a type of sphingolipid; the ceramide pathway of exosomal secretion has been shown to affect the apoptosis of cancer cells. But the role of this pathway in MM cell function, exosome function and miR regulation remains unknown. In this study, we showed that C6 ceramide (an exogenous ceramide supplement, 1.25-40 $\mu \mathrm{mol} / \mathrm{L}$ ) dose-dependently inhibited the proliferation and promoted the apoptosis in human MM OPM2 cell line, which were associated with elevated caspase 3/9 and PARP cleavage. We also found that C6 ceramide (5-20 $\mu \mathrm{mol} / \mathrm{L})$ dose-dependently stimulated exosome secretion and increased exosomal levels of tumor-suppressive miRs (miR 202, miR 16, miR 29b and miR 15a). Of note, exosomes from C6 ceramide-treated OPM2 cells could influence the proliferation and apoptosis of the recipient OPM2 cells, which correlated with increased tumor-suppressive exosomal miRs. In contrast, GW4869 (a ceramide inhibitor, 5-20 $\mathrm{mol} / \mathrm{L}$ ) exerted the opposite effects on the regulation of MM function, exosome secretion and miR levels in MM exosomes. However, exosomes from GW4869-treated OPM2 cells had no effect on these miRs and the survival of targeted OPM2 cells. Taken together, our findings reveal that the ceramide pathway modulates MM survival, probably directly via the caspase pathway and indirectly via exosomal miR mechanisms.
\end{abstract}

Keywords: multiple myeloma; exosomes; microRNAs; ceramide pathway; apoptosis; caspase pathway; C6 ceramide; GW4869; OPM2 cell line

Acta Pharmacologica Sinica (2018) 39: 561-568; doi: 10.1038/aps.2017.118; published online 31 Aug 2017

\section{Introduction}

Multiple myeloma (MM) is characterized by uncontrolled monoclonal plasma cell proliferation and is the second most common hematological malignancy. It accounts for nearly $20 \%$ of deaths related to hematological malignancy ${ }^{[1,2]}$. MM has a low 5-year survival rate of approximately $40 \%$ and is frequently refractory to traditional therapies ${ }^{[3,4]}$. As such, indepth investigation of the cell biology of MM is urgently needed in order to develop novel chemotherapeutic targets for MM.

Mounting evidence has shown that cell-released exosomes play an essential role in intercellular communication through the transfer of bioactive molecules such as proteins, mRNAs

\footnotetext{
\# These authors contributed equally to this work.

* To whom correspondence should be addressed.

E-mail lj0jingliu@163.com

Received 2017-03-20 Accepted 2017-06-18
}

and microRNAs (miRs) ${ }^{[5,6]}$. Extracellular exosomes are emerging as potential therapeutic strategies and diagnostic biomarkers for various diseases, including cardiovascular diseases, neurological disorders and cancers ${ }^{[5,7-9]}$. Despite an incomplete understanding of the mechanisms of exosome secretion, ceramide (a type of sphingolipid) has been well characterized as regulating exosome secretion under both physiological and pathological conditions ${ }^{[10,11]}$. Interestingly, ceramide has also been shown to regulate growth, proliferation and apoptosis in multiple cancers, such as leukemia, colon carcinoma and ovarian cancer $^{[12-14]}$. Ceramide C6 (C6-cer), as an exogenous ceramide supplement, can significantly induce apoptosis in different cancer cell lines ${ }^{[15-17]}$. On the other hand, the ceramide inhibitor GW4869 can remarkably decrease exosome generation and can promote human hepatocellular cancer cell proliferation $^{[18,19]}$. These observations suggest that the ceramide pathway plays a pivotal role in exosome secretion and the 
functions of cancer cells. However, the role of the ceramide pathway in the cellular and exosomal functions of MM has not been explored.

Tumor-suppressive miRs have been suggested to be involved in regulating MM survival. It was previously reported that the over-expression of $\mathrm{miR} 29 \mathrm{~b}$ induces apoptosis and caspase 3 activation in MM cells ${ }^{[20]}$ and that miR $15 \mathrm{a} / 16$ can promote MM apoptosis by suppressing the expression of the anti-apoptosis protein Bcl- $2^{[21]}$. Moreover, miR 202 can inhibit proliferation and can induce apoptosis in MM cells via regulating $\mathrm{B}$-cell activating factors $(\mathrm{BAFF})^{[22]}$. Of note, exosomes are the means to transfer miRNAs from donor cells to recipient cells, representing a novel method of intercellular communication $^{[23]}$. Moreover, the ceramide pathway has been shown to induce the apoptosis of metastatic prostate cancer cells by triggering the secretion of tumor-suppressive miRs ${ }^{[24]}$. However, whether the ceramide pathway regulates the cellular and exosomal miRs of MM remains unclear.

In this study, we examined the effects of the ceramide pathway on the cellular and exosomal functions of MM. For our mechanistic investigation, we analyzed the possible links between the caspase-dependent pathway and tumor-suppressive miRs (miR 202, miR 16, miR 29b and miR 15a).

\section{Materials and methods Cell culture}

Multiple myeloma (OPM2 cell line) was generously gifted by Dr Feng-huang ZHAN (University of Iowa). Cells were cultured in RPMI-1640 medium (HyClone, USA) with 10\% fetal bovine serum (FBS) (HyClone, USA) and maintained at $37^{\circ} \mathrm{C}$ with $5 \% \mathrm{CO}_{2}$ and $95 \%$ humidity. GW4869 or C6-cer (Sigma, USA) was routinely stored at $-80^{\circ} \mathrm{C}$ as a $5 \mathrm{mmol} / \mathrm{L}$ stock suspension in DMSO. Immediately prior to use, the suspension was solubilized by the addition of phosphate-buffered saline (PBS) and diluted into a working solution. The suspension was completely mixed and warmed to $37^{\circ} \mathrm{C}$ until clear.

\section{MTT assay of cell proliferation}

OPM2 cells $\left(4 \times 10^{4}\right.$ cells $\left./ \mathrm{mL}\right)$ were treated with different concentrations of C6-cer or GW4869 and seeded in 96 well plates. After $24 \mathrm{~h}$ of incubation, cell viability was assessed by adding $1.0 \mathrm{mg} / \mathrm{mL}$ of Thiazolyl Blue Tetrazolium Bromide (Sigma Aldrich, USA) to the medium. After $4 \mathrm{~h}$ of incubation, the plate was centrifuged and the supernatant was removed. The pellets were solubilized with $100 \mu \mathrm{L} /$ well DMSO. The absorbance of the samples against a background control (medium alone) was measured at $570 \mathrm{~nm}$ with a microliter plate reader (BioTek Instruments, USA). All experiments were performed in five replicates.

\section{Apoptosis detection by Hoechst 33342 staining}

A Hoechst 33342 staining kit (BD, USA) was used to detect cell apoptosis. Cells were treated with C6-cer or GW4869 for $24 \mathrm{~h}$. Then, cells were harvested, washed, and resuspended in $1 \times$ binding buffer according to the protocol provided in the kit. In the next step, cells were stained with Hoechst 33342 at
$1 \mathrm{mg} / \mathrm{mL}$ for $15 \mathrm{~min}$. Then, cells were washed with PBS twice and imaged under a fluorescence microscope.

\section{Exosome isolation, detection and quantification Collection}

Exosomes were prepared from culture supernatants of OPM2 cells by differential centrifugations. Briefly, the cell medium was first collected and centrifuged at $300 \times g$ for $15 \mathrm{~min}$, followed by centrifugation at $2000 \times g$ for $30 \mathrm{~min}$ to remove cells and cell debris. The cell-free culture medium was centrifuged at $20000 \times g$ for $70 \mathrm{~min}$ and then ultracentrifuged at $170000 \times g$ for $1.5 \mathrm{~h}$ to pellet the exosomes. The pelleted exosomes were resuspended with $20 \mathrm{~nm}$ filtered PBS (Whatman, USA).

\section{Particle size and concentration analysis}

Exosomes were resuspended in PBS, and the particle size and concentration were measured by using the Nanoparticle Tracking Analysis System 300 (NTA300, Malvern Instruments, UK). The NTA300 method has been recognized as an easy-touse, rapid and reproducible platform for exosome characterization $^{[8]}$. In this study, diluted suspensions containing exosomes were loaded into the sample chamber. The light scatter mode of the tracking system used camera filter 1 . Three videos of typically $30 \mathrm{~s}$ in duration were taken, with a frame rate of 30 frames per second. Data were analyzed by NTA 3.0 software (Malvern Instruments, USA), which was optimized to first identify and then to track each particle on a frame-byframe basis ${ }^{[8]}$.

\section{Immunofluorescence staining}

To identify the efficiency of exosome uptake, exosomes were labeled with PKH26 (Invitrogen, USA). Briefly, $30 \mu \mathrm{g}$ of exosomes were re-suspended in $1 \mathrm{~mL}$ of Diluent $\mathrm{C}$. Then, $4 \mu \mathrm{L}$ of PKH26 were mixed with Diluent $\mathrm{C}$ prior to staining. The PKH26 suspension was mixed with the exosome suspension and incubated for $4 \mathrm{~min}$, and protected from light. The staining reaction was stopped by the addition of an equal volume of $1 \%$ BSA and centrifuged at $170000 \times g$ for $90 \mathrm{~min}$ at $4{ }^{\circ} \mathrm{C}$. Then, pelleted exosomes were washed with PBS and centrifuged at $170000 \times g$ for $90 \mathrm{~min}$ at $4{ }^{\circ} \mathrm{C}$. The exosomes were suspended in $200 \mu \mathrm{L}$ of supplemented RMPI-1640 medium and cultured with a confluent layer of OPM2 cells. Following co-incubation for $24 \mathrm{~h}$, OPM2 cells were washed with PBS, stained with DAPI for $5 \mathrm{~min}$, washed with PBS and fixed with $4 \%$ formaldehyde. Images of exosome uptake by OPM 2 cells were acquired with an LSM710 laser scanning microscope (Carl Zeiss, Germany).

\section{Protein extraction and Western blot analysis}

Whole-cell lysates and total exosomal proteins were prepared using RIPA buffer $(50 \mathrm{mmol} / \mathrm{L}$ Tris- $\mathrm{HCl} \mathrm{pH} 7.4,150 \mathrm{mmol} / \mathrm{L}$ $\mathrm{NaCl}, 1 \% \mathrm{NP} 40,0.5 \%$ sodium deoxycholate, $0.1 \%$ sodium dodecyl sulfate). We electrophoretically separated $50 \mu \mathrm{g}$ total protein on a $4 \%-12 \%$ SDS-acrylamide gel (Thermo Fisher Scientific, USA). Western blot analyses were performed with the following primary antibodies: anti- $\beta$-actin, anti-caspase 3 , anti- 
cleaved caspase 3, anti-caspase 9, anti-cleaved caspase 9, antiPARP, anti-cleaved PARP (1:1000, Cell Signaling Technology, USA), and the corresponding anti-mouse and anti-rabbit peroxidase-linked secondary antibodies (1:10000; Cell Signaling Technology, USA). The signals were visualized by the ECL Prime Western Blotting Detection Reagent (Advansta, USA).

\section{RNA extraction and quantitative real-time PCR of miRs}

Total RNA was isolated using Trizol Reagent (Invitrogen, USA) according to the manufacturer's instructions. The purified RNA was subjected to reverse transcription reactions using the PrimeScript ${ }^{\mathrm{TM}}$ RT reagent kit (Takara, Japan). Quantitative real-time PCR (qRT-PCR) with SYBR Premix Ex Taq II Mix (Takara, Japan) was used to evaluate transcript levels; U6 was used as an internal control. All of the primer sequences are reported in Supplementary Table S1. qRT-PCR assays were performed in the StepONEPlus Real-Time PCR system (Thermo Fisher Scientific, USA) and the relative expression was calculated using the comparative $2^{-\Delta \Delta C t}$ method.

\section{Statistical analysis}

Statistical significance was calculated in Microsoft Excel using Student's $t$-test (one-sided) or ANOVA, as appropriate. The generated data displayed a normal distribution with similar variance, and analysis was performed assuming equal variance.

\section{Results}

The effect of the ceramide pathway on MM proliferation

To determine the effect of the ceramide pathway on MM proliferation, OPM2 cells were treated with C6-cer or GW4869 at increasing concentrations $(0,1.25,2.5,5,10,20,40 \mu \mathrm{mol} / \mathrm{L})$ for $24 \mathrm{~h}$ and then proliferation was assessed with the MTT assay. As shown in Figure 1A, the proliferation of OPM2 cells was dose-dependently inhibited by C6-cer. By contrast, the cell proliferation rate was increased after GW4869 treatment (Figure 1B). The inhibition of cell proliferation was only significant when the concentration of GW4869 was $40 \mu \mathrm{mol} / \mathrm{L}$ or higher.

The effect of the ceramide pathway on MM apoptosis and the caspase pathway

MM cell apoptosis in this study was evaluated by Hoechst 33342 staining assay (Figure 2A). The apoptosis of MM cells was increased after C6-cer treatment $(20 \mu \mathrm{mol} / \mathrm{L})$ compared with controls $(20.5 \% \pm 1.72 \%$ vs $3.7 \% \pm 1.54 \%, P<0.05$; $n=3$, Figure $2 \mathrm{~B})$. There was no significant difference in $\mathrm{MM}$ apoptosis following treatment with GW4869 $(20 \mu \mathrm{mol} / \mathrm{L})$. To explore the potential mechanism underlying the effect of the ceramide pathway on MM apoptosis, we assessed the expression levels of caspase 3/9, PARP and their cleaved forms (Figure 2C). There was no change in the total expression of caspase 3/9 or PARP. However, cleaved caspase $3 / 9$ and PARP were significantly increased after C6-cer treatment (Figure 2D). These results indicated that the activation of the ceramide pathway promotes MM apoptosis, probably though the caspase pathway.
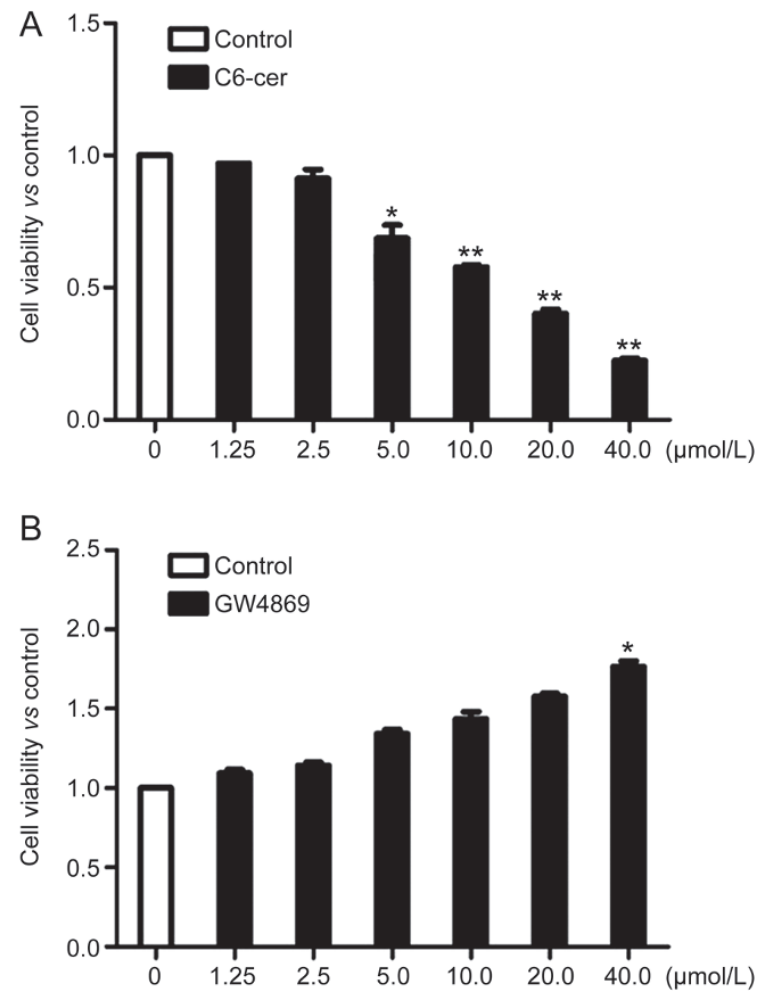

Figure 1. Effect of ceramide pathway on the proliferation of MM cells. (A) The effect of C6-cer on the proliferation of MM cells. (B) The effect of GW4869 on the proliferation of MM cells. Error bars represent the SEM for 3 independent experiments. ${ }^{*} P<0.05,{ }^{* *} P<0.01$, compared to Control, $n=5$ /group.

The effect of the ceramide pathway on exosome release from MM cells

To examine the effects of the ceramide pathway on exosome release from MM cells, OPM2 cells were treated with C6-cer or GW4869 separately at increasing concentrations $(0,5.0,10$, $20 \mu \mathrm{mol} / \mathrm{L})$. Then, the size and concentration were analyzed using the NTA 300. The size of OPM2 exosomes derived from C6-cer or GW4869 treatment mainly distributed from $40 \mathrm{~nm}$ to $200 \mathrm{~nm}$ (Figure 3A, 3C). C6-cer dose-dependently increased the number of exosomes released. The release of exosomes was increased nearly five-fold at the concentration of 20 $\mu \mathrm{mol} / \mathrm{L}$ compared with controls (Figure 3B). By contrast, GW4869 gradually decreased exosome secretion by approximately $80 \%$ at a concentration of $20 \mu \mathrm{mol} / \mathrm{L}$ (Figure 3D).

The effect of the ceramide pathway on the cellular and exosomal miR levels of MM cells

To further confirm the role of the ceramide pathway on the levels of cellular and exosomal tumor-suppressive miRs in MM, OPM2 cells were treated with $20 \mu \mathrm{mol} / \mathrm{L}$ C6-cer or GW4869 separately for $24 \mathrm{~h}$. The levels of miRs in cells and exosomes were measured using qRT-PCR. As shown in Figure $4 A-4 D$, we detected decreased levels of miR 202, miR 16, miR 29b and miR 15a in MM cells and increased levels of these miRs in exosomes after C6-cer treatment. The opposite 

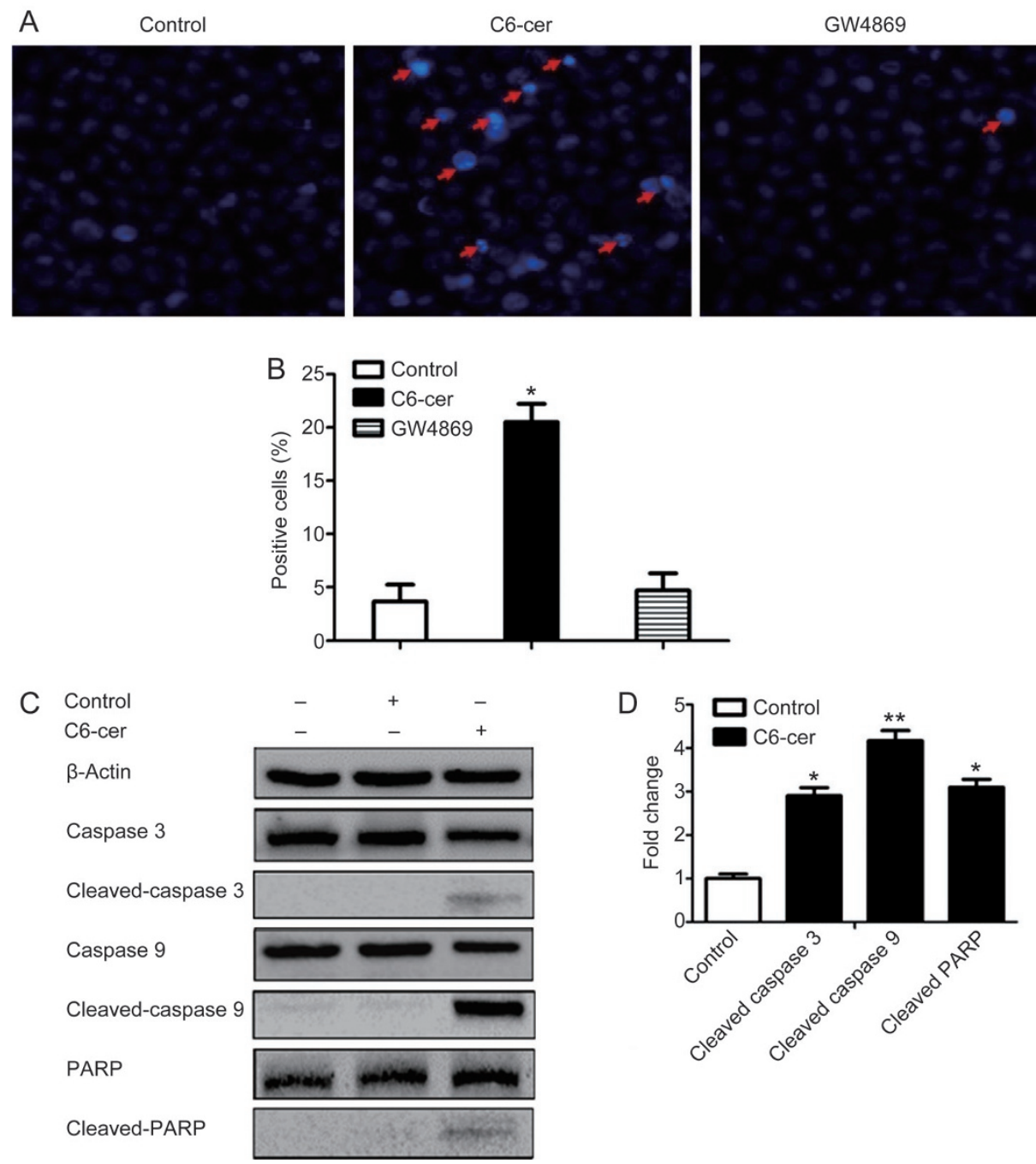

Figure 2. Effect of ceramide pathway on the apoptosis of MM cells. (A) Representative pictures of Hoechst 33342 stain in MM cells which were treated with DMSO only (control), $10 \mu \mathrm{mol} / \mathrm{L}$ C6-cer and $10 \mu \mathrm{mol} / \mathrm{L}$ GW4869 for $24 \mathrm{~h}$; (B) Summarized data of positive cells in Hoechst 33342 stain assay, shown as mean \pm SEM; $n=3$ /group ( ${ }^{*} P<0.05$, compared to control); (C) Western blots with anti-caspase 3 , anti-cleaved caspase 3 , anti-caspase 9 , anticleaved caspase 9, anti-PARP and anti-cleaved PARP antibodies. $\beta$-Actin is used as normalization control for the total lysate. The data shown were representatives of three independent experiments that gave similar results; (D) Summarized data of Western blots (white bar: control; black bar: C6cer), shown as mean \pm SEM; $n=3$ /group. ${ }^{*} P<0.05,{ }^{* *} P<0.01$ compared to control.

changes were found in the miR levels in MM cells and exosomes following treatment with GW4869 (Figure 4E-4H).

The effect of the ceramide pathway on exosome functions and the miR levels of targeted MM cells

We isolated MM exosomes after treatment with C6-cer (exosomes $^{\mathrm{C} \text {-cer }}$ ) or GW4869 (exosomes ${ }^{\mathrm{GW} 4869}$ ) using differential centrifugation. As shown in Figure 5A, Western blot analysis showed that the isolated MM exosomes were rich in CD63 and lacked Annexin V. The efficiency of exosome uptake was confirmed $24 \mathrm{~h}$ later using the PKH26 stain (Supplementary Figure S1). After protein quantification, OPM2 cells were treated with $80 \mu \mathrm{g} / \mathrm{mL}$ exosomes ${ }^{\mathrm{C} 6-\mathrm{cer}}$ or exosomes ${ }^{\mathrm{GW} 4869}$ for $48 \mathrm{~h}$. The results showed that the proliferation of OPM2 cells was significantly inhibited by exosomes ${ }^{\mathrm{C} 6 \text {-cer }}$ treatment (Figure
5A) in a time-dependent manner. In addition, exosomes ${ }^{\mathrm{C} 6-\mathrm{cer}}$ increased the apoptosis of MM cells $(3.2 \% \pm 1.3 \%$ vs $9.1 \% \pm 1.9 \%$, $P<0.05 ; n=3)$. However, exosomes ${ }^{\mathrm{C} 6-\mathrm{cer}}$ had no effect on the expression of cleaved caspase 3/9 or PARP. Of note, the levels of these tumor-suppressive miRs were significantly altered in exosomes ${ }^{\mathrm{C} 6-\mathrm{cer}}$ treated MM cells. As shown in Figure 5, exosomes ${ }^{\mathrm{GW} 4869}$ had no effect on cell proliferation, apoptosis or the miR concentrations of the recipient MM cells.

\section{Discussion}

In the present study, we demonstrated that the ceramide pathway can affect MM cells at several functional levels, including proliferation, apoptosis and exosomal, and revealed that these effects of the ceramide pathway are associated with the caspase pathway and several tumor-suppressive miRs, including 

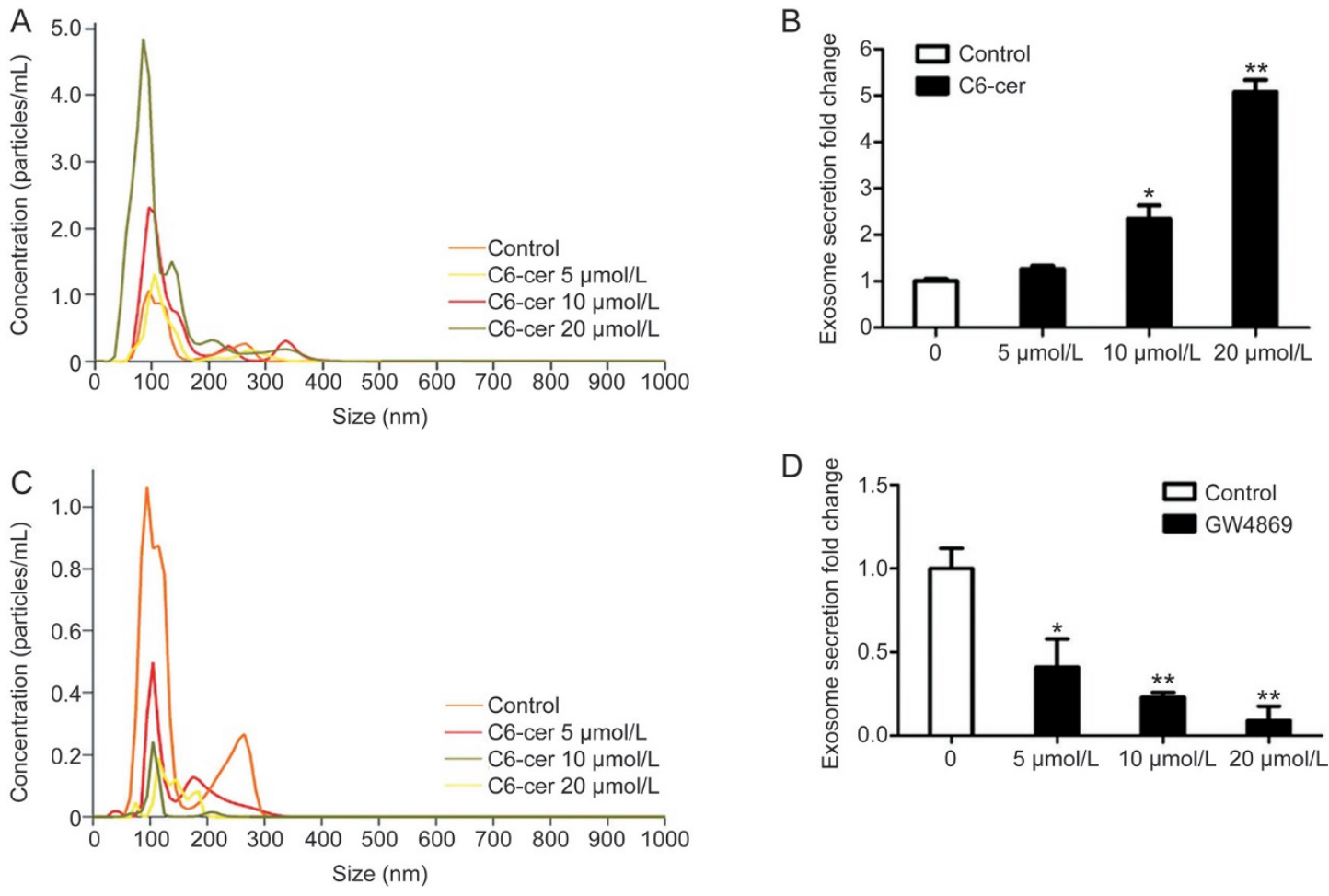

Figure 3. The effect of ceramide pathway on exosome secretion of MM cells. (A and C) Representative plots showing the size/concentration distribution of the secreted exosomes of C6-cer and GW4869 treated by using Nano Tracking System 300; (B and D) C6-cer and GW4869 dose-dependently inhibited/stimulated exosome release from MM cells. ${ }^{*} P<0.05,{ }^{* *} P<0.01$, compared to Control, $n=3 /$ group.
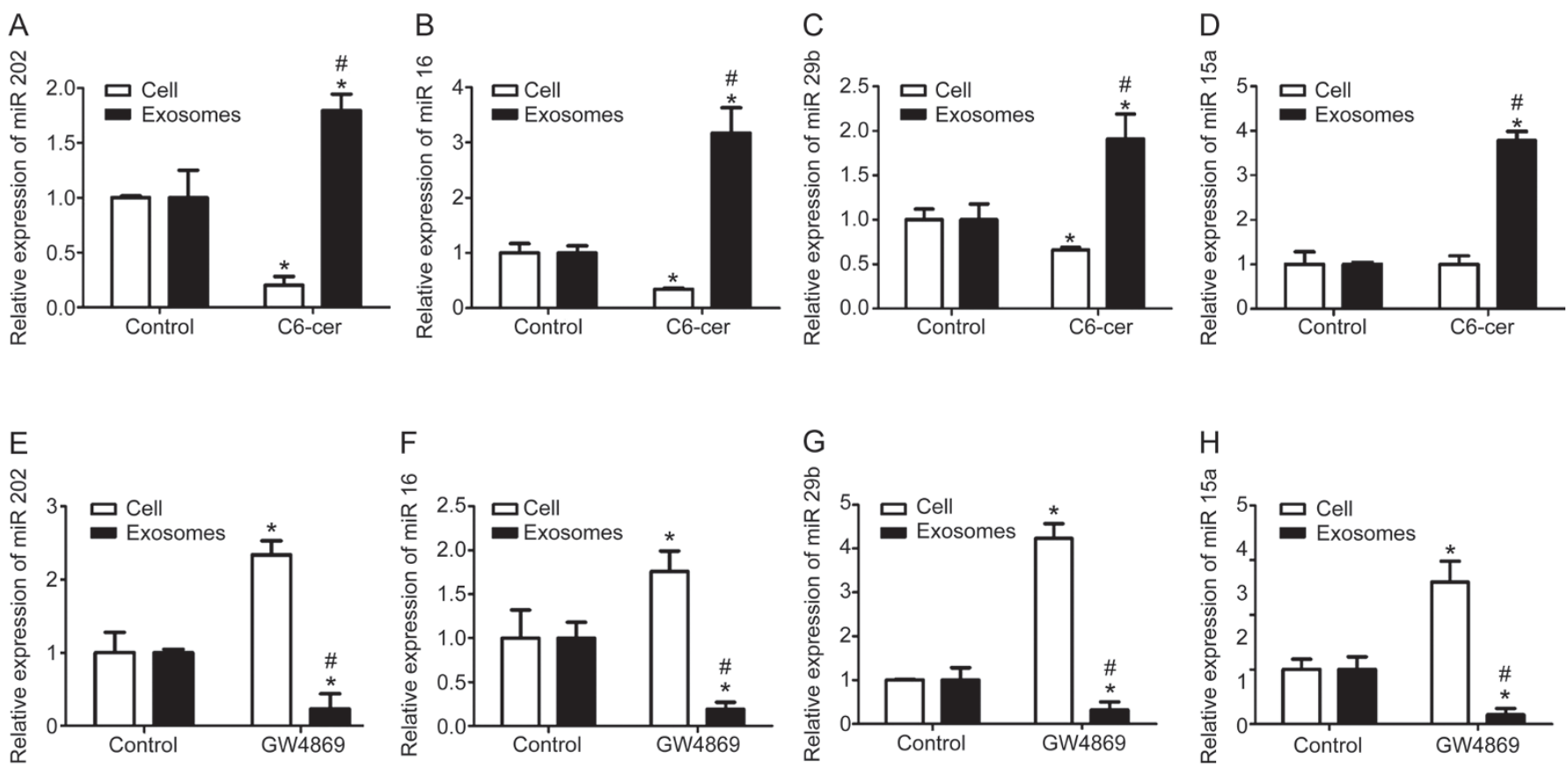

Figure 4. Ceramide pathway modulates the levels of several tumor-suppressive miRs in MM cells and their released exosomes. Samples of cell pellets and exosomes after C6-cer treated (A-D) and GW4869 treated (E-H) were prepared, RNA was extracted, and qRT-PCR analyses were performed. U6 small nuclear RNA (snRNA) was used as internal controls. Data were shown as the mean \pm SEM. ${ }^{*} P<0.05$, compared to control; ${ }^{*} P<0.05$, compared to cells; $n=3$ /group. 

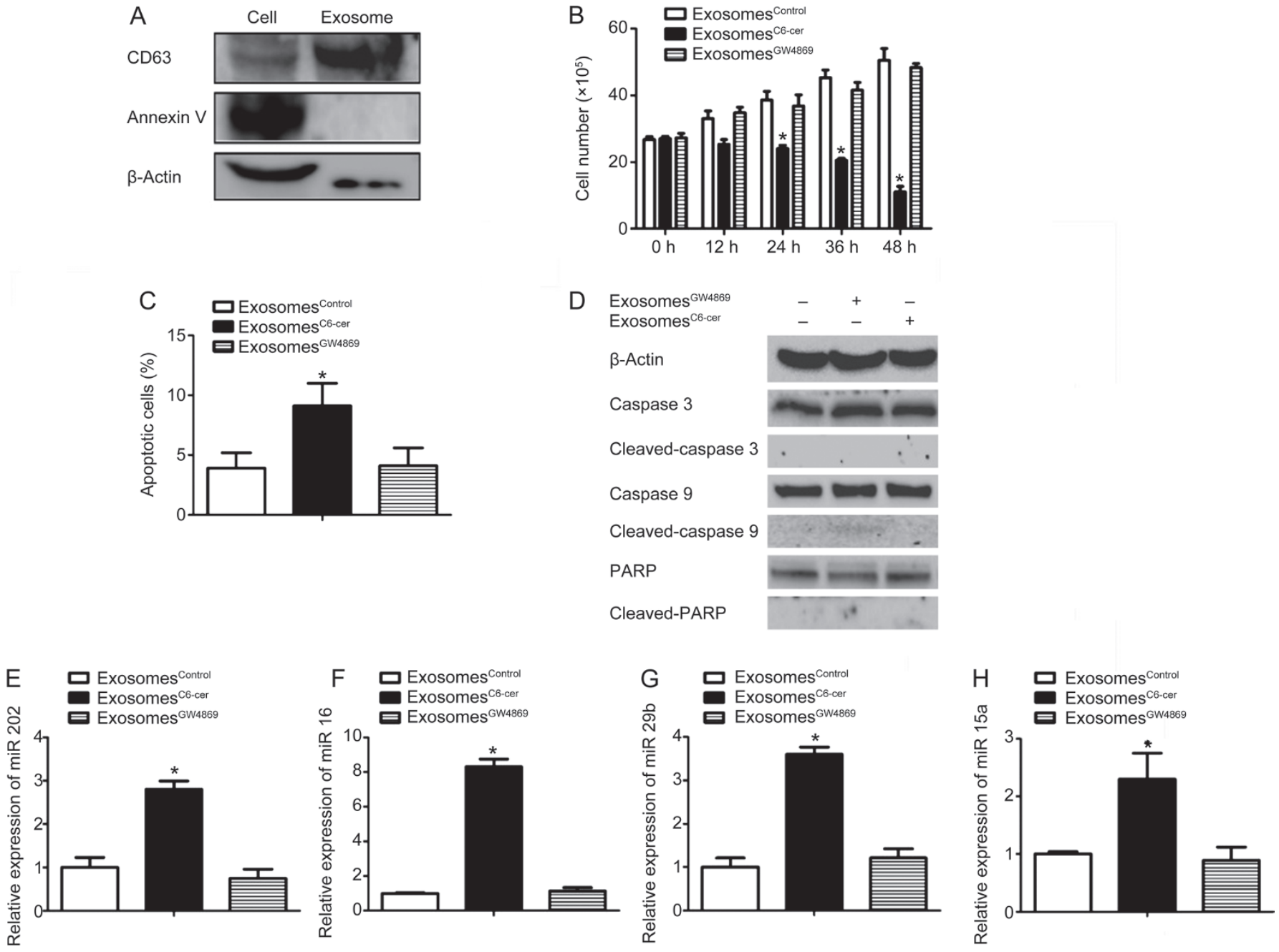

Figure 5. The effect of ceramide pathway on the exosome functions and miR levels of targeting MM cells. (A) Markers for MM exosomes that were examined by using Western blot. (B) The effects of exosomes ${ }^{\text {C6-cer }}$ and exosomes ${ }^{\text {GW4869 }}$ on MM cell growth; (C) The effect of exosomes ${ }^{\text {C6-cer }}$ or exosomes $^{\mathrm{GW} 4869}$ on MM apoptosis by using Hoechst 33342 stain assay; (D) Expressions of caspase pathway after the treatment of exosomes ${ }^{\mathrm{Cb}-\mathrm{cer}}$ or exosomes ${ }^{\mathrm{GW} 4869}$; (E, F) The levels of miR 202, miR 16, miR 29b and miR 15 a were measured by qRT-PCR in MM cells treated by exosomes ${ }^{\mathrm{C}-\mathrm{cer}}$ or

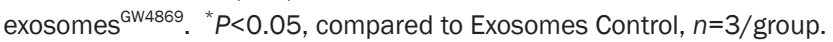

miR 146a, miR 29b, miR 15a and miR 16 (Figure 6).

Increasing evidence suggests that the ceramide pathway plays a crucial role in the proliferation and apoptosis of different cancer cells, such as colon carcinoma ${ }^{[25]}$, ovarian ${ }^{[14]}$ and breast cancer ${ }^{[15]}$. In this study, the roles of the ceramide pathway in the proliferation and apoptosis of MM cells were determined by using C6-cer and GW4869 to modulate the activity of this pathway, as previously reported ${ }^{[15,16,26]}$. We found that C6-cer dose-dependently $(1.25-40 \mu \mathrm{mol} / \mathrm{L})$ inhibited MM proliferation (5\%-80\%), whereas GW4869 promoted proliferation (1- to 2-fold) in the same concentration range. These results are consistent with previous reports showing the effects of C6-cer and GW4869 on the proliferation of breast cancer cells ${ }^{[26,27]}$. In addition, we found that C6-cer increased the apoptotic ratio of MM cells, which is similar to a previous study that demonstrated that C6-cer activates caspase-dependent apoptotic death in melanoma cells ${ }^{[28]}$. For the mechanistic analysis, we measured the levels of caspase 3/9 and PARP cleavage in MM cells. We found that C6-cer increased the expression of caspase 3/9 and PARP cleavage. However, we did not detect any significant effect of GW4869 on MM apoptosis. The failure of GW4869 to influence MM apoptosis is explainable as we did not observe any changes in the expression of caspase $3 / 9$ or PARP cleavage. On the other hand, MM cells had a low basal apoptotic rate (approximately $4 \%$ ) and low levels of caspase $3 / 9$ and PARP cleavage, which could mask the effects of GW4869. Therefore, our data suggest that the ceramide pathway can modulate MM proliferation and apoptosis, probably through the mitochondrial caspase pathway.

The ceramide pathway has also been demonstrated to participate in the modulation of exosome release in several cancer cells, including breast cancer ${ }^{[15]}$ and prostate cancer ${ }^{[24]}$. However, whether it regulates the release of MM exosomes remains unknown. In this study, we showed that the activa- 


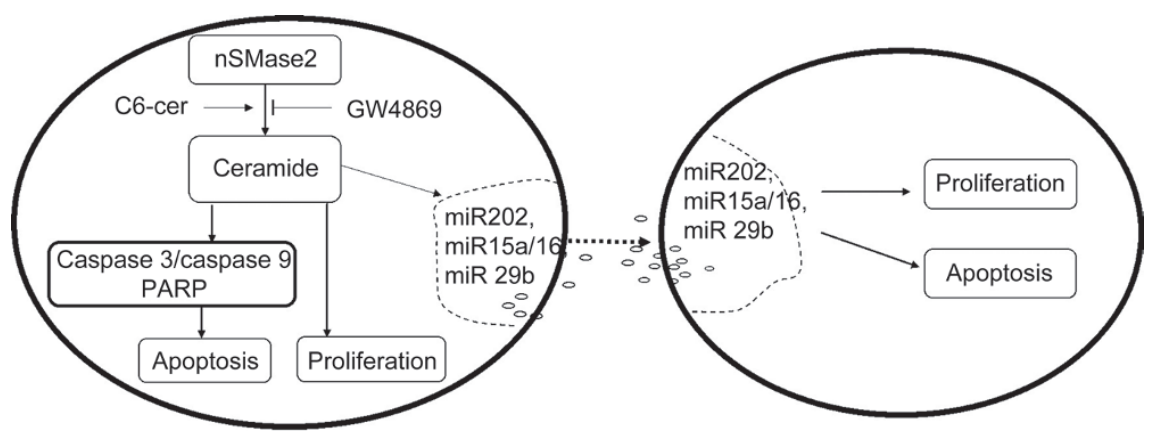

Figure 6. Ceramide pathway affects MM survival directly and indirectly via exosomal miRs.

tion of the ceramide pathway increased exosome release up to five fold, while the inhibition of this pathway decreased exosome release by approximately $80 \%$. Our results indicate that the ceramide pathway is involved in regulating the release of MM exosomes. Additionally, the ceramide pathway has been shown to regulate tumor-related miRs in prostate cancer cells, as well as their released exosomes ${ }^{[24]}$. Previous studies have shown that miR 29b, miR 15a/16 and miR 202 are involved in MM proliferation and apoptosis ${ }^{[20,21,29]}$. Therefore, we also examined the effect of the ceramide pathway on the cellular and exosomal levels of these tumor-suppressive miRs. Our data showed that the activation of the ceramide pathway decreased the levels of these miRs in MM cells, but increased their levels in MM exosomes. On the other hand, inhibition of the ceramide pathway had contrasting effects. Our results suggest that the ceramide pathway plays an important role in the control of these miR levels in MM cells and exosomes. Of note, the alterations in intracellular miRs induced by C6-cer/ GW4869 could not explain the changes in MM function (proliferation and apoptosis). This discrepancy might be explained by the fact that C6-cer/GW4869 affects other pathways that overwhelm the effects of changes in tumor-suppressive miRs. Indeed, we discovered that C6-cer activated the caspase pathway in MM cells with upregulated caspase 3/9 and PARP cleavage by 4-fold. However, whether C6-cer induced cell apoptosis and exosome secretion are dependent on the caspase pathway requires further confirmation with a caspase inhibitor.

Cancer cell-derived exosomes have "paracrine" functions by communicating with surrounding cells through the transfer of bioactive molecules (miRs, mRNAs and proteins), which implicates exosomes in the proliferation and apoptosis of cancer cells ${ }^{[23,30]}$. A previous study showed that MM exosomes could stimulate the secretion of cytokines from stromal cells to favor MM cell growth and migration ${ }^{[31]}$. However, it is unclear whether MM exosomes could perform these "paracrine" effects on neighboring MM cells. We isolated MM exosomes after separate C6-cer and GW4869 treatment, defined as exosomes ${ }^{\mathrm{C} 6 \text {-cer }}$ and exosomes ${ }^{\mathrm{GW} 4869}$, to determine their "paracrine" effects on untreated MM cells. Our results showed that exosomes ${ }^{\mathrm{C} 6 \text {-cer }}$ inhibited proliferation and increased apoptosis in MM cells. To better understand the mechanism, we focused on caspase 3/9 and PARP, as well as these miRs. Interestingly, we did not detect any changes in cleaved caspase 3/9 and PARP, suggesting that the caspase pathway was not responsible for the effects of exosomes ${ }^{\mathrm{C}-\mathrm{cer}}$ in recipient $\mathrm{MM}$ cells. However, we found that exosomes ${ }^{\mathrm{C} 6 \text {-cer }}$ increased the levels of miR 202, miR 29b, miR 15a and miR 16 in treated MM cells. Therefore, a possible explanation for the function of exosomes ${ }^{\mathrm{C} 6 \text {-cer }}$ might be related to the increased levels of these tumor-suppressive miRs in exosomes ${ }^{\mathrm{C} 6-\mathrm{cer}}$ treated MM cells, although their downstream effectors, such as Bcl-2 and Mcl-1, remain to be elucidated. In addition, we found that exosomes ${ }^{\mathrm{GW} 4869}$ had no effect on the proliferation and apoptosis of recipient MM cells. A reasonable explanation for this finding is that the levels of miRs are unchanged in exosomes ${ }^{\text {GW4869 }}$ treated MM cells as exosomes ${ }^{\text {GW4869 }}$ have very low levels of these miRs. Moreover, in agreement with our observation that GW4869 had no effect on the caspase pathway of MM, exosomes ${ }^{\text {GW4869 }}$ did not affect this pathway in exosomes ${ }^{\text {GW4869 }}$ treated MM cells. Taken together, our data indicate that exosomes $^{\mathrm{C} 6-\mathrm{cer}}$ inhibits proliferation and induces apoptosis in MM cells, probably by regulating these tumor-suppressive miRs in the targeted MM cells.

In conclusion, we identified important roles for the ceramide pathway in the proliferation, apoptosis and exosome functions of MM cells. Of note, the ceramide pathway modulates MM apoptosis, presumably by regulating the caspase-dependent pathway. Exosomes related to the ceramide pathway activated in MM cells inhibit proliferation and increase apoptosis in recipient MM cells, which correlated with the increased levels of exosomal miR 202, miR 29b, miR 15a and miR 16. This study provides a novel understanding of MM biology that will have significant implications for the development of novel therapeutic and biomarker strategies.

\section{Acknowledgements}

This study was supported by the National Natural Science Foundation of China (№ 81470361 and 81670203) and TianQing Program (№ B16416-20160824) to Jing LIU; the CSC Scholarship (№ 201706370184) and the Innovative Research Funds for the Central South University to Qian CHENG (№ 2016zzts151).

\section{Author contribution}

Jing LIU conceived the study and participated in its design and coordination; Qian CHENG performed the majority of 
the study and wrote the manuscript; Xin LI and Feng-huang ZHAN helped to draft the manuscript and participated in the English language editing; Min DONG and Yue WANG performed the data analysis.

\section{Supplementary information}

Supplementary information is available at the website of the Acta Pharmacologica Sinica.

\section{References}

1 Raab MS, Podar K, Breitkreutz I, Richardson PG, Anderson KC. Multiple myeloma. Lancet 2009; 374: 324-39.

2 Sabattini E, Bacci F, Sagramoso C, Pileri SA. WHO classification of tumours of haematopoietic and lymphoid tissues in 2008: an overview. Pathologica 2010; 102: 83-7.

3 Brenner H, Gondos A, Pulte D. Recent major improvement in longterm survival of younger patients with multiple myeloma. Blood 2008; 111: 2521-6.

4 Chung $\mathrm{C}$. Role of immunotherapy in targeting the bone marrow microenvironment in multiple myeloma: an evolving therapeutic strategy. Pharmacotherapy 2017; 37: 129-43.

5 Wang Z, Chen JQ, Liu JL, Tian L. Exosomes in tumor microenvironment: novel transporters and biomarkers. J Transl Med 2016; 14: 297.

6 Munson P, Shukla A. Exosomes: potential in cancer diagnosis and therapy. Medicines (Basel) 2015; 2: 310-27.

7 Wu CY, Du SL, Zhang J, Liang AL, Liu YJ. Exosomes and breast cancer: a comprehensive review of novel therapeutic strategies from diagnosis to treatment. Cancer Gene Ther 2017; 24: 6-12.

8 Wang J, Guo R, Yang Y, Jacobs B, Chen S, Iwuchukwu I, et al. The novel methods for analysis of exosomes released from endothelial cells and endothelial progenitor cells. Stem Cells Int 2016; 2016: 2639728.

9 Wang Y, Cheng Q, Liu J, Dong M. Leukemia stem cell-released microvesicles promote the survival and migration of myeloid leukemia cells and these effects can be inhibited by microRNA34a overexpression. Stem Cells Int 2016; 2016: 9313425.

10 Trajkovic K, Hsu C, Chiantia S, Rajendran L, Wenzel D, Wieland F, et al. Ceramide triggers budding of exosome vesicles into multivesicular endosomes. Science 2008; 319: 1244-7.

11 Wang G, Dinkins M, He Q, Zhu G, Poirier C, Campbell A, et al. Astrocytes secrete exosomes enriched with proapoptotic ceramide and prostate apoptosis response 4 (PAR-4): potential mechanism of apoptosis induction in Alzheimer disease (AD). J Biol Chem 2012; 287: 21384-95.

12 Zhang J, Alter N, Reed JC, Borner C, Obeid LM, Hannun YA. Bcl-2 interrupts the ceramide-mediated pathway of cell death. Proc Natl Acad Sci U S A 1996; 93: 5325-8.

13 Coe GL, Redd PS, Paschall AV, Lu C, Gu L, Cai H, et al. Ceramide mediates FasL-induced caspase 8 activation in colon carcinoma cells to enhance FasL-induced cytotoxicity by tumor-specific cytotoxic $T$ lymphocytes. Sci Rep 2016; 6: 30816.

14 Kitatani K, Usui T, Sriraman SK, Toyoshima M, Ishibashi M, Shigeta S, et al. Ceramide limits phosphatidylinositol-3-kinase C2beta-controlled cell motility in ovarian cancer: potential of ceramide as a metastasissuppressor lipid. Oncogene 2016; 35: 2801-12.

15 Kong JN, He Q, Wang G, Dasgupta S, Dinkins MB, Zhu G, et al. Guggulsterone and bexarotene induce secretion of exosomeassociated breast cancer resistance protein and reduce doxorubicin resistance in MDA-MB-231 cells. Int J Cancer 2015; 137: 1610-20.

16 Ji C, Yang B, Yang YL, He SH, Miao DS, He L, et al. Exogenous cellpermeable 66 ceramide sensitizes multiple cancer cell lines to Doxorubicin-induced apoptosis by promoting AMPK activation and mTORC1 inhibition. Oncogene 2010; 29: 6557-68.

17 Zhang F, Yang J, Li H, Liu M, Zhang J, Zhao L, et al. Combating rituximab resistance by inducing ceramide/lysosome-involved cell death through initiation of CD20-TNFR1 co-localization. Oncoimmunology 2016; 5: e1143995.

18 Iguchi Y, Eid L, Parent M, Soucy G, Bareil C, Riku Y, et al. Exosome secretion is a key pathway for clearance of pathological TDP-43. Brain 2016; 139: 3187-201.

19 Essandoh K, Yang L, Wang X, Huang W, Qin D, Hao J, et al. Blockade of exosome generation with GW4869 dampens the sepsis-induced inflammation and cardiac dysfunction. Biochim Biophys Acta 2015; 1852: 2362-71.

20 Zhang YK, Wang H, Leng Y, Li ZL, Yang YF, Xiao FJ, et al. Overexpression of microRNA-29b induces apoptosis of multiple myeloma cells through down regulating $\mathrm{Mcl}-1$. Biochem Biophys Res Commun 2011; 414: 233-9.

21 Li Y, Zhang B, Li W, Wang L, Yan Z, Li H, et al. MiR-15a/16 regulates the growth of myeloma cells, angiogenesis and antitumor immunity by inhibiting Bcl-2, VEGF-A and IL-17 expression in multiple myeloma. Leuk Res 2016; 49: 73-9.

22 Shen X, Guo Y, Yu J, Qi J, Shi W, Wu X, et al. miRNA-202 in bone marrow stromal cells affects the growth and adhesion of multiple myeloma cells by regulating $B$ cell-activating factor. Clin Exp Med 2016; 16: 307-16.

23 Thind A, Wilson C. Exosomal miRNAs as cancer biomarkers and therapeutic targets. J Extracell Vesicles 2016; 5: 31292.

24 Kosaka N, Iguchi H, Yoshioka Y, Takeshita F, Matsuki Y, Ochiya T. Secretory mechanisms and intercellular transfer of microRNAs in living cells. J Biol Chem 2010; 285: 17442-52.

25 Coe GL, Redd PS, Paschall AV, Lu C, Gu L, Cai H, et al. Ceramide mediates FasL-induced caspase 8 activation in colon carcinoma cells to enhance FasL-induced cytotoxicity by tumor-specific cytotoxic $T$ lymphocytes. Sci Rep 2016; 6: 30816.

26 Flowers M, Fabrias G, Delgado A, Casas J, Abad JL, Cabot MC. C6-ceramide and targeted inhibition of acid ceramidase induce synergistic decreases in breast cancer cell growth. Breast Cancer Res Treat 2012; 133: 447-58.

27 Wu M, Harvey KA, Ruzmetov N, Welch ZR, Sech L, Jackson K, et al. Omega-3 polyunsaturated fatty acids attenuate breast cancer growth through activation of a neutral sphingomyelinase-mediated pathway. Int J Cancer 2005; 117: 340-8.

28 Jiang F, Jin K, Huang S, Bao Q, Shao Z, Hu X, et al. Liposomal C6 ceramide activates protein phosphatase 1 to inhibit melanoma cells. PLoS One 2016; 11: e159849.

29 Zhang Y, Shen XJ, Wu XH, Cong H, Ni HB, Ju SQ, et al. miR-202 contributes to sensitizing $\mathrm{MM}$ cells to drug significantly via activing JNK/SAPK signaling pathway. Zhonghua Xue Ye Xue Za Zhi 2016; 37: 987-92.

30 Zhang L, Pan L, Xiang B, Zhu H, Wu Y, Chen M, et al. Potential role of exosome-associated microRNA panels and in vivo environment to predict drug resistance for patients with multiple myeloma. Oncotarget 2016; 7: 30876-91.

31 De Veirman K, Wang J, Xu S, Leleu X, Himpe E, Maes K, et al. Induction of miR-146a by multiple myeloma cells in mesenchymal stromal cells stimulates their pro-tumoral activity. Cancer Lett 2016; 377: $17-24$. 